Retraction

\title{
Retraction Note: Correlation Between Anthropometric, Physical Fitness Traits, Lung Capacity, and Success of Iranian Elite Greco-Roman Wrestlers
}

\author{
Vahid Ziaee ${ }^{1,2,{ }^{*}}$ \\ ${ }^{1}$ Sports Medicine Research Center, Neuroscience Institute, Tehran University of Medical Sciences, Tehran, Iran \\ ${ }^{2}$ Division of Pediatric Rheumatology, Children's Medical center, Pediatrics Center of Excellence, Tehran University of Medical Sciences, Tehran, Iran \\ "Corresponding author: Sports Medicine Research Center, Neuroscience Institute, Tehran University of Medical Sciences, Tehran, Iran. Email: ziaeev@gmail.com
}

Received 2019 March 15; Accepted 2019 May 19.

This retracts the article "RETRACTED ARTICLE: Correlation Between Anthropometric, Physical Fitness Traits, Lung Capacity, and Success of Iranian Elite Greco-Roman Wrestlers".

Following the author's declaration received to the Asian Journal of Sports Medicine on 15 March 2019 (Ticket \#378448) and in line with COPE flowchart (http://bit.ly/2fCptzz ) about duplicate publications, the editor-in-chief of the journal decided to retract the mentioned article from the publishing process. Based on a written document, the corresponding author (Hossein Taheri Chadorneshin) declared that it has been previously published by the same authors in a Turkish journal [International Journal of Science Culture and Sport (IntJSCS)]. Most of the text and all the tables are the same as in the article that was published in Turkish journal. The author agreed to this retraction.

Kind Regards,

Editor-in-Chief,

Ziaee, Vahid,

Tehran, IR Iran 